\title{
Author Correction: Structural basis for the regulation of inositol trisphosphate receptors by $\mathrm{Ca}^{2+}$ and $\mathrm{IP}_{3}$
}

Navid Paknejad (D) and Richard K. Hite (1)

Correction to: Nature Structural \& Molecular Biology https://doi.org/10.1038/s41594-018-0089-6, published online 16 July 2018.

In the version of this article initially published, one of the PDB codes associated with the study was listed in the data availability statement as 6DBC but should have been 6DRC. The error has been corrected in the HTML and PDF versions of the article. 